\title{
FATORES QUE AFETAM A SAZONALIDADE DA CPUE NA PESCARIA BRASILEIRA DO BONITO LISTRADO (KATSUWONUS PELAMIS) E IMPLICAÇÕES PARA A AVALIAÇÃO DA PESCARIA
}

\author{
H. A. ANDRADE \\ UNIVALI/CTTMar, Caixa Postal 360, 88302-202, Itajaí-SC \\ E-mail: humber@cttmar.univali.br
}

\begin{abstract}
RESUMO
Há grandes oscilações sazonais da captura por unidade de esforço (CPUE) na pescaria de vara e isca-viva do bonito listrado (Katsuwonus pelamis), realizada na costa do Brasil. A CPUE é um índice que reflete as variações do coeficiente de capturabilidade e da abundância da população que é explotada pela pesca. No entanto, as variações sazonais da abundância, ao contrário das interanuais, não são importantes para a avaliação de uma pescaria. Sob esse prisma de avaliação da pescaria, a análise das causas das oscilações cíclicas da CPUE, pode ser tomada como equivalente à análise das causas das variações sazonais do coeficiente de capturabilidade (q). Os principais fatores identificados como determinantes dessas oscilações de q foram: recrutamento, migração, feições e processos oceanográficos, visualização dos cardumes, variação na disponibilidade de alimento, e variação no número e dimensão dos cardumes. A análise da sazonalidade de q serve também de laboratório para investigação sobre as variações interanuais do parâmetro. Tais variações podem tornar os dados de CPUE inúteis para a avaliação da pescaria. Os problemas com as variações interanuais de q podem estar relacionados com a escolha da unidade de esforço a ser usada. A necessidade de estimativas de um índice de procura de cardumes (ex: área varrida), emerge como um dos conhecimentos fundamentais para a construção de uma unidade de esforço adequada.
\end{abstract}

Palavras-chave: CPUE, coeficiente de capturabilidade, vara e isca-viva, bonito listrado, Katsuwonus pelamis.

\section{FACTORS AFFECTING THE SEASONAL VARIATION OF CPUE AND THE ASSESSMENT OF SKIPJACK TUNA (KATSUWONUS PELAMIS) FISHERY ON BRAZILIAN WATERS}

\begin{abstract}
In the Brazilian skipjack tuna (Katswonus pelamis) pole and line fishery, large seasonal oscillations of catch per unit of effort (CPUE) has been observed. As an index, CPUE should reflect variation in the coefficient of catchability (q) as well as in the abundance of the exploited populations. In opposition to the interannual variations, seasonal variations in the population abundance are unimportant in the conceptual framework of most fishery assessment models. Therefore, a plausible simplification would be assume the analysis of factors affecting seasonal oscillations of CPUE as equivalent to the analysis of the seasonal oscillations of the coefficient of catchability. In the Brazilian skipjack tuna fishery, recruitment, migration, oceanographic circulation, sighting of schools, variation in the food availability and variations in the number and dimension of the schools have been identified as the main factors affecting seasonal oscillations in q. The study of these oscillations is important in order to identify and understand also the interannual oscillations in q which may prevent the use of CPUE data in the fishery assessment. Problems of interannual oscillations in $q$ are generally related to the choice of the unit of effort. In the case of the skipjack tuna fishery, the estimation of an index reflecting the search effort (e.g. swept area), seems to be essential for the establishment of a more suitable unit of effort to be used in its stock assessment.
\end{abstract}

Key words: CPUE, Catchability Coefficient, Pole an Line, Skipjack Tuna, Katsuwonus pelamis. 


\section{A PESCA DO BONITO LISTRADO (KATSUWONUS PELAMIS)}

A pescaria do bonito listrado (Katsuwonus pelamis) (Scombridae: Thunnini) apresenta em todo o mundo grandes variações sazonais quanto a área de ocorrência e a captura por unidade de esforço (CPUE) (Willians, 1970; Sund et al., 1981; Tameishi \& Shinomiya, 1989; Andrade, 1996). A pescaria no Brasil, iniciada em 1979 com o uso de vara e isca-viva, não é uma exceção. A freqüência de ocorrência das pescarias e a CPUE, são baixas no inverno, quando a pescaria é realizada principalmente na costa sudeste, e altas no verão, quando a pescaria é feita basicamente na costa sul (Fig. 1) (Vilela, 1990; Vilela \& Castello, 1993; Andrade, 1996; Andrade \& Garcia, 1999).

As variações sazonais da CPUE são em grande parte, um reflexo de oscilações cíclicas do coeficiente de capturabilidade. Além das variações sazonais naturais da disponibilidade de um recurso pelágico, outros fatores técnicos, como por exemplo variações sazonais da dimensão do campo de visada utilizado na procura de cardumes, podem também influenciar 0 coeficiente de capturabilidade. Considerações a respeito de fatores que influenciam no coeficiente de capturabilidade auxiliam em uma decisão sobre se o esforço de pesca e a CPUE atuais são adequados, e também indicam lacunas de conhecimentos para que estas quantidades possam ser melhor estimadas.

\section{CPUE}

A CPUE usualmente adotada na pescaria do bonito no Brasil, tem como unidade de esforço, dias de pesca ou de mar. Entende-se como dia de pesca, qualquer dia em que ocorreu pelo menos uma tentativa de captura após o cardume ter sido avistado e abordado. A abordagem do cardume consiste na aproximação do barco e na liberação de isca (pequenos peixes pelágicos vivos) para atração do peixe. Quando o bonito é atraído e começa a se alimentar, é iniciada a pescaria com o uso de linhas, anzóis e caniços, normalmente confeccionados de bambu.

Os registros de mapas de bordo disponíveis para a pesca do SE-S do Brasil apresentam apenas a atividade "pescaria". Não é indicado o tempo despendido na procura antes da pesca. O número de dias registrados como dias de procura (ou seja quando houve procura e não foi encontrado nenhum cardume) é extremamente baixo, comprovando a baixa freqüência do evento ou mesmo uma subestimativa do mesmo. Portanto, no cálculo da CPUE com dias de pesca ou mesmo com dias de mar como unidade de esforço, não é levado em conta de forma clara, o tempo de procura ou mesmo a área varrida na procura. Assim, a CPUE é somente a captura realizada, condicionado ao fato de que o cardume foi encontrado, e portanto, não é um índice adequado da abundância relativa e da densidade do recurso na área de pesca.

A disponibilidade do bonito para a pesca é proporcional à dificuldade de visualizar, atrair, manter o cardume no costado do bar-

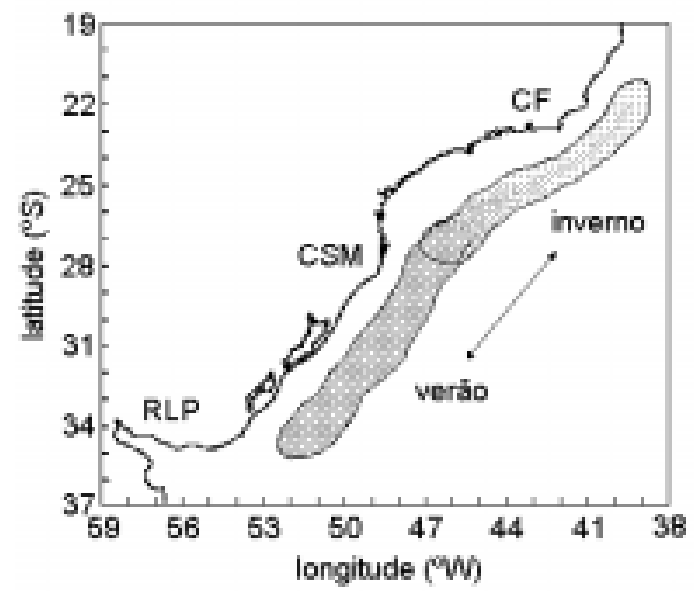

Figura 1 - Representação esquemática das principais áreas de pesca de bonito listrado (Katsuwonus pelamis) na costa do Brasil. (CF) Cabo Frio; (CSM) Cabo de Santa 
co, e realizar a captura. Portanto, a CPUE usualmente calculada, mesmo com todos os problemas metodológicos, pode ser vista ainda como um índice da disponibilidade do recurso para a pesca. Sob esse prisma, conclui-se que o bonito está bem mais disponível para a pesca no verão (Vilela, 1990; Vilela \& Castello, 1993; Andrade \& Garcia, 1999). As causas desse padrão estão provavelmente relacionadas aos fatores: (a) recrutamento; (b) emigração e variação do número de cardumes; (c) dimensão dos cardumes; (d) visualização dos cardumes; (e) feições oceanográficas (i.e. frentes); e (f) variações na disponibilidade de alimentos e do comportamento de alimentação. Avanços tecnológicos que aumentam o poder de pesca não são considerados uma vez que não são componentes cíclicos.

\section{RECRUTAMENTO}

O bonito tem um comportamento reprodutivo normalmente denominado de oportunista. O que significa que havendo condições adequadas é possível que haja alguma desova e que há quase sempre alguma fêmea em estágio avançado de maturação (Cayré \& Farrugio, 1986). Apesar disso, há um foco principal de desova nas costas norte e nordeste, nas imediações da primavera e do verão (Matsuura, 1985; Castello \& Habiaga, 1989). Este fato não implica na formação de uma coorte na concepção usual da palavra, mas resulta na formação de um pulso reprodutivo relativamente bem definido. Aparentemente o recrutamento desses indivíduos jovens para a pesca, ocorre nas imediações do verão (Campos \& Andrade, 1997). A disponibilidade desse novo e numeroso grupo de indivíduos poderia ser um dos fatores que contribuem para o incremento das CPUEs no verão, seja através de aumento do número ou mesmo da dimensão dos cardumes. $\mathrm{O}$ recrutamento é um componente das variações na abundância do recurso. Portanto, o fato da CPUE sofrer o efeito do recrutamento para a pesca não é problemático, pois conceitualmente a CPUE deve mesmo ser um reflexo da abundância e de seus componentes. No entanto, variações no número e/ou dimensão dos cardumes podem ter outras causas que não o recrutamento e/ou variações na abundância.

\section{EMIGRAÇÃO E VARIAÇÃO DO NÚMERO DE CARDUMES}

Uma causa possível para as baixas CPUEs que ocorrem nas imediações do inverno, seria que nesse período, seguindo a tendência geral de migração para o norte, uma parcela importante do recurso ocupe uma área onde a frota não atua (ao norte de 195) (Fig. 1). De fato, existem relatos de que há uma concentração considerável de cardumes de bonito na região de Abrolhos no inverno (Matsuura, com. pess.), mas não houve até então o desenvolvimento de uma pescaria.

Apesar de ser um aspecto importante, uma possível migração de parte do recurso para fora da área tradicional de pesca, pode não ter uma relação direta com a CPUE usualmente adotada ( $\mathrm{kg} / \mathrm{dia}$ de pesca). Uma vez que a CPUE é reflexo da captura obtida dado que o cardume foi encontrado, caso a pescaria diária ocorresse sempre sobre um único cardume, a CPUE sazonal seria independente do número de cardumes disponíveis. Assim, emigrações para fora da área de pesca só dificultariam o encontro do cardume, mas não iriam afetar a CPUE, considerando a forma como esse índice é calculado no caso dessa pescaria.

Atualmente a hipótese de que a pescaria diária ocorra quase que estritamente sobre só um cardume tem pouca credibilidade, pois há relatos de pescadores, contrários a essa posição. Caso seja confirmado que há normalmente tempo hábil para se pescar sobre mais de um cardume em um único dia, a CPUE poderia então ser afetada pelo número de cardumes disponíveis, independente da interferência desse número no 
tempo de procura. Para um melhor entendimento da CPUE, restaria então investigar se o número de cardumes avistados e abordados por dia varia sazonalmente e é maior no verão. Essa questão é fundamental para o estabelecimento de uma boa unidade de esforço.

\section{DIMENSÃO DOS CARDUMES}

A possibilidade de que a biomassa dos cardumes seja menor no inverno/maior no verão é outra hipótese que poderia justificar a variações sazonais da CPUE. Além do já mencionado recrutamento nas imediações do verão, variações na dimensão média dos cardumes na área de pesca, poderiam ter como causa, a emigração. Como conseqüência do deslocamento da maior parte do recurso para o norte no inverno (Fig. 1), restariam ainda na costa sudeste somente os grupos de cardumes marginais, menos numerosos e com menor dimensão do que ao norte, no centro de distribuição do recurso. Discussões acerca da possibilidade de que populações de peixes que formam cardumes apresentem tal organização de grupos são encontradas em Lebedev (1967). Investigações sobre possíveis variações sazonais da dimensão média dos cardumes disponíveis (e.g. acústicas ou aéreas) deveriam ser feitas no futuro.

\section{VISUALIZAÇÃO DOS CARDUMES}

A probabilidade dos cardumes serem encontrados por visualização direta, poderia variar em função do período do ano. É importante notar que essa hipótese está também vinculada à veracidade de que em um dia possam ser abordados mais de um cardume. A discussão que vem a seguir é feita considerando que essa suposição é satisfeita.

Algumas características corroboram com a possibilidade de que a visualização seja facilitada no verão. Há evidências de que a termoclina seja mais rasa nessa época do ano, no sul (Castello \& Möller, 1977; Lima \&
Castello, 1995), onde o bonito está mais disponível para a pesca. Uma termoclina rasa e pronunciada poderia restringir os cardumes às menores profundidades aumentando a probabilidade dos mesmos aflorarem na superfície, e serem visualizados. A diminuição da profundidade da termoclina no verão, está relacionada à penetração da Água Central do Atlântico Sul (ACAS) pelo fundo da plataforma continental (Castello \& Möller, 1977; Matsuura, 1986; Castro Filho et al., 1987; Lima \& Castello, 1995).

As condições climatológicas e da superfície do mar podem também ser fatores que facilitam a visualização de cardumes no verão. Segundo relato dos pescadores, as condições do mar no inverno são normalmente piores que no verão. A visualização do cardume pode ser por exemplo dificultada se o vento é forte e/ou as vagas tem maior altura.

Aves podem ser indicadores importantes da presença de cardumes de bonito (e.g. Chiaradia, 1991), porém é pouco provável que variações cíclicas na efetividade/presença de indicadores visuais (e.g. aves e mamíferos marinhos) sejam importantes para a explicação da sazonalidade da CPUE.

Investigações esclarecedoras sobre se a CPUE é afetada por processos e variáveis oceanográficas (i.e. altura do mar) que possam facilitar ou dificultar a visualização de cardumes são necessárias, mas podem ser mais difíceis de executar do que parece. Por exemplo, pode ser relativamente fácil mostrar que o campo de visada é menor no inverno. No entanto, pode ser difícil esclarecer se a CPUE é baixa no inverno porque a visualização é dificultada ou porque os cardumes são realmente menos abundantes. Discussões relevantes sobre correlações entre variáveis bióticas e abióticas, incluindo possíveis soluções metodológicas para a investigação de tais problemas tem sido propostas nos últimos anos (Walters \& Collie, 1988; Perry et al., 1994; Smith \& Page, 1996; Andrade \& Garcia, 1999). 


\section{FEIÇÕES OCEANOGRÁFICAS}

Além das já mencionadas feições oceanográficas que podem facilitar a visualização (i.e. termoclinas rasas), outras como por exemplo frentes e vórtices, poderiam favorecer a agregação de cardumes e portanto resultar em maiores CPUEs caso a pesca ocorra sobre mais de um cardume por dia.

A agregação de atuns junto a frentes oceanográficas tem sido amplamente sugerida (Laurs \& Lynn, 1977; Laurs et al., 1984; Fiedler \& Bernard, 1987; Tameishi \& Shinomiya, 1989; Power \& May, 1991). Entretanto, no caso da pescaria do bonito na costa do Brasil, ainda não há resultados conclusivos sobre essa questão. Resultados iniciais indicam que no Brasil a formação de frentes oceanográficas superficiais na área de pesca é mais freqüente do outono à primavera, enquanto que no verão as CPUEs são altas apesar da baixa incidência de frentes (Andrade, 1996; Andrade \& Garcia 2000). Portanto, atualmente a ocorrência de frentes não pode ser considerado um fator que resulta no incremento da vulnerabilidade do bonito no verão, influenciando a sazonalidade da CPUE.

\section{VARIAÇÕES NA DISPONIBILIDADE DE ALIMENTOS E DO COMPORTAMENTO DE ALIMENTAÇÃO}

A captura depende de que o bonito seja atraído pela isca lançada na água. Se o engodo funciona, a pescaria é iniciada. A persistência do cardume, alimentando-se por um período prolongado, antes de se dispersar ou abandonar as imediações da embarcação, é também crucial. A possibilidade de que no inverno o cardume possa estar menos disposto a se alimentar da isca oferecida, permanecendo por pouco tempo nas imediações da embarcação, deve então ser considerada. A investigação dessa questão passa provavelmente por uma análise de conteúdo estomacal (incluindo Índices de Repleção - IR), e pela coleta de informações sobre o tempo de permanência do cardume no costado do barco e sobre a variação sazonal da abundância de presas potenciais. Até o momento os dados são parcos e pouco informativos quanto a possibilidade de que haja variações sazonais significativas do IR (ver Vilela, 1990).

\section{CONSIDERAÇÕES FINAIS}

A procura de hipóteses para explicar a sazonalidade da CPUE passa por uma dissecção do problema e pela identificação dos pontos críticos. Todos os fatores discutidos ( $\mathrm{n}^{\circ}$ de peixes por cardumes, recrutamento, atração, $\mathrm{n}^{\circ}$ de cardumes, etc) estão efetivamente vinculados à maior ou menor vulnerabilidade do bonito para a pesca, e portanto ao coeficiente de capturabilidade (q) (Fig. 2). Dentre as informações atualmente inexistentes e importantes para esclarecimento de como a maioria dos fatores destacados afeta a vulnerabilidade do bonito, a necessidade de estimativas de um índice do esforço de pesca relativo à procura dos cardumes (e.g. área varrida), é sem dúvida a mais relevante.

A lacuna de um "índice de esforço de procura" constitui um problema não só na análise das variações sazonais, mas também nas análises das oscilações interanuais da CPUE. A CPUE atualmente usada (captura por dias de pesca ou de mar) é aparentemente inadequada, uma vez que ela não seria representativa de abundâncias e densidades relativas.

Uma primeira tentativa de solucionar o problema poderia ser feita com a modificação dos mapas de bordo para incluir informações diárias sobre o horário e o tempo de permanência na gávea, do pescador que procura o cardume. Outra informação relevante seria a altura das gáveas das embarcações. Quanto ao uso das informações pretéritas, há a possibilidade de que correções possam ser feitas através de informações que poderiam ser geradas através de um programa de ob- 
servadores de bordo. Seria possível chegar por exemplo a estimativas do percentual médio gasto na procura em viagens, para cada estação do ano e área de pesca. A partir dessa informação, os vícios dos dados pretéritos poderiam ser amenizados.

Mesmo quando as informações aparentemente relevantes estão contabilizadas, a utilidade da CPUE tem sido alvo de muita discussão em virtude de problemas com a unidade de esforço, com possíveis alterações do coeficiente de capturabilidade, e mesmo com incertezas sobre a forma da relação entre a CPUE e a abundância (e.g. Clarck, 1985; Hilborn \& Walters, 1992).

A questão primordial é se é possível estabelecer uma relação coerente entre a CPUE anual e a abundância, e se ela pode então ser interpretada como um índice relativo. Desde que previamente identificadas e conhecidas as informações necessárias para a estruturação de um esforço de pesca (ex: fatores expostos na Fig. 2), modelos lineares generalizados são no momento a técnica mais promissora para a análise e estimativa de índices de abundância relativa (Hilborn \& Walters, 1992). Uma tentativa de ajuste de tais modelos à dados da pescaria de bonito na costa do Brasil, utilizando a unidade de esforço usual e problemática, "dias de pesca", foi feita recentemente (Menezes et al.,

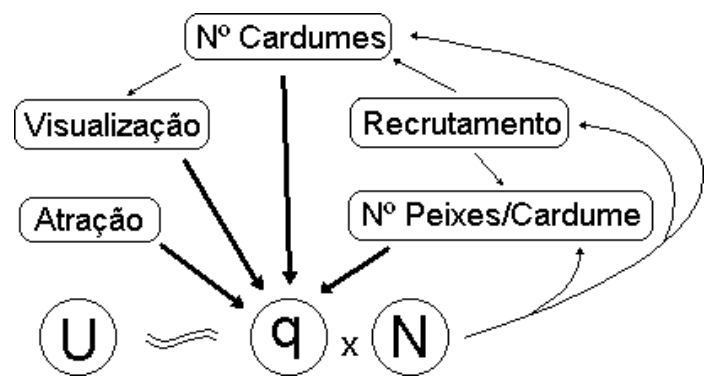

Figura 2 - Diagrama dos fatores envolvidos na relação entre a abundância/densidade do bonito listrado (Katsuwonus pelamis) e a CPUE atualmente calculada no caso da pescaria brasileira.
2000). A CPUE não mostrou nenhuma variação significativa desde o início da pescaria até o momento atual, mesmo que tenha havido variações claras do esforço de pesca utilizado (dias de pesca). Esse padrão não é comum para uma pescaria comercial estabelecida a quase 20 anos. $O$ esperado seria que ocorressem quedas e incrementos da CPUE em função de aumentos e diminuições do esforço de pesca. Portanto, antes desses resultados iniciais serem usados para auxiliar decisões e ações de manejo pesqueiro, certamente devem ser estruturados novos modelos após a definição de uma unidade de esforço mais adequada.

\section{REFERÊNCIAS BIBLIOGRÁFICAS}

Andrade, H. A. 1996. Distribuição, abundância relativa e migração do Katsuwonus pelamis (Scombridae) em relação à temperatura superficial do mar e à dinâmica oceanográfica na costa sudeste - sul do Brasil. Tese de Mestrado. Universidade do Rio Grande, RS, Brasil. 148 pp.

Andrade, H. A. \& C. A. E. Garcia. 1999. Skipjack Tuna Fishery in Relation to Sea Surface Temperature in the Southern Brazilian Coast. Fish. Oceanogr. 8:245254.

Andrade, H. A. \& C. A. E. Garcia. 2000. Skipjack Tuna Fishery in Relation to Environmental Variations in the Southwestern South Atlantic. (manuscrito).

Campos, R. O. \& H. A. Andrade. 1997. Estudo da Migração e do Recrutamento do Bonito Listado (Katsuwonus pelamis) Através da Estrutura de Comprimento. Anais do III Seminário Integrado de Iniciação Científica. Setembro de 1997. Itajaí-SC. $47 \mathrm{p}$.

Castello, J. P. \& R. P. Habiaga. 1989. The Skipjack Tuna Fishery in the Southern Brazil. SCRS/88/27, ICCAT Coll. Vol. Sci. Pps., 30(1):6-19. 
Castello, J. P. \& O. O. Möller. 1977. Sobre as condições oceanográficas no Rio Grande do Sul. Atlântica. 2(2):25-110.

Castro Filho, B. M., L. B. Miranda \& S. Y. Miyao. 1987. Condições hidrográficas na plataforma continental ao largo de ubatuba: variações sazonais e em média escala. Bolm Inst. Oceanogr., S. Paulo. 35(2):135-151.

Cayré, P. \& H. Farrugio. 1986. Biologie de la Reproduction du Listao (Katsuwonus pelamis) de l'Océan Atlantique. Proc. ICCAT Conf. Intern. Skipjack Year Prog., 252-272 p.

Chiaradia, A. 1991. Interação entre aves marinhas e cardumes de Bonito Listado (Katsuwonus pelamis) na costa sul do Brasil. Atlântica 13(1):115-118.

Clark, C. W. 1985. Bioeconomic Modelling and Fisheries Management. Wiley Interscience. $291 \mathrm{pp}$.

Fiedler, P.C. and Bernard, H.J. 1987. Tuna aggregation and feeding near fronts observed in satellite imagery. Cont. Shelf Res. 7(8):871-881.

Hilborn, R. \& C. J. Walters. 1992. Quantitative Fisheries Stock Assessment. Chapman and Hall, New York. 570 pp.

Laurs, M. \& R. J. Lynn, R.J. 1977. Seasonal migration of north pacific albacore, Thunnus alalunga, into North American coastal waters: distribution, relative abundance, and association with transition zone waters. Fish. Bull. 75(4):795-823.

Laurs, R.M.; Fiedler, P.C. \& D. R. Montgomery. 1984. Albacore tuna catch distributions relative to environmental features observed from satellites. Deep-Sea Res. 31:1085-1099.

Lebedev, N. V. 1967. Elementary Populations of Fish. Wiener Bindery Ltd., Jerusalem. 224 pp.

Lima, I. D. \& J. P. Castello. 1995. Distribution and Abundance of South-west Atlantic Anchovy Spawners (Engraulis anchoita) in Relation to Oceanographic Processes in the Southern Brazilian Shelf. Fish. Oceanogr. 4(1):1-16.

Matsuura, Y. 1985. Distribution and Abundance of Skipjack Larvae Off the Coasts of Brazil. Proc. ICCAT Conf. Intern. Skipjack Year Prog., 285-289 p.

Matsuura, Y. 1986. Contribuição ao estudo da estrutura oceanográfica da região sudeste entre Cabo Frio (RJ) e Cabo de Santa Marta Grande (SC). Ciência e Cultura. 38(8):1439-1450.

Meneses L., J. H., C. F. Lin \& A. S. Menezes. 2000. Preliminary Results of Standardized Catch Rates for Skipjack Tuna (Katsuwonus pelamis) from the Brazilian Baitboat Fishery through 1998. SCRS/99/ 69. Coll. Vol. Sci. Pps., 50(3):000-000. (no prelo).

Perry, R. I., M. Stocker \& J. Fargo. 1994. Environmental Effects on the Distributions of Groundfish in Hecate Strait, British Columbia. Can. J. Fish. Aquat. Sci. 51:1401-1409.

Power, J. H. \& L. N. May Jr. 1991. Satellite Observed Sea-Surface Temperatures and yellowfin Tuna Catch and Effort in the Golf of Mexico. Fish. Bull., 89:429-439.

Smith, S. J. \& F. H. Page. 1996. Associations Between Atlantic Cod (Gadus morhua) and Hydrographic Variables: Implications for the Management of the $4 \mathrm{VsW}$ Cod Stock. ICES J. mar. Sci. 53:597-614.

Sund, P. N., M. Blackburn \& F. Willians. 1981. Tunas and their Environment in the Pacific Ocean: A Review. Oceanogr. Mar. Biol. Ann. Rev., 19:443-512.

Tameishi, H. \& H. Shinomiya. 1989. Formation of Southbound Skipjack Fishing Grounds and its Discriminant Prediction Off Touhoku Sea Area. Nippon Suisan Gakkaishi, 55(4):619-625.

Vilela, M. J. A. 1990. Idade, Crescimento, Alimentação e Avaliação do Estoque de Bonito Listado, Katsuwonus pelamis (Scombridae: Thunnini), Explorado na Região Sudeste-Sul do Brasil. Tese de 
Mestrado. Universidade do Rio Grande, RS, Brasil. 81 pp.

Vilela, M. J. A. \& J. P. Castello. 1993. Dinamica Poblacional del Barrilete (Katsuwonus pelamis) Explotado en la Region Sudeste-Sur del Brasil en el Periodo 1980-1986. Fren. Marit. 9:29-35.

Walters, C. J. \& J. S. Collie. 1988. Is Research on Environment Factors Useful to Fisheries Management? Can. J. Fish. Aquat. Sci. 45:1848-1854.
Willians, F. 1970. Sea Surface Temperature and the Distribution and Aparent Abundance of Skipjack Tuna (Katsuwonus pelamis) in the Eastern Pacific Ocean 1951-1968. Inter-Am. Trop. Tuna Comm. Bull., 15:229-281. 